\title{
Aspectos destacados de las Guías de la Sociedad Europea de Cardiología sobre cardiología deportiva y ejercicio en pacientes con enfermedad cardiovascular
}

\author{
Dra. María Victoria Ramos
}

\section{Introducción}

La actividad física regular, incluyendo el ejercicio sistemático, forma parte de la estrategia de tratamiento de múltiples enfermedades cardiovasculares (ECV) y se asocia con reducción de la mortalidad (tanto cardiovascular [CV] como por todas las causas). El estilo de vida actual, que favorece el sedentarismo y la obesidad (con el consecuente aumento en ECV), obliga a mayor énfasis en la promoción de actividad física y ejercicio regular, debiendo ser prioridad para las sociedades científicas.

Pese a sus evidentes beneficios, el ejercicio puede, paradójicamente, desencadenar muerte súbita cardíaca (MSC) en individuos con ECV, particularmente en aquellos previamente sedentarios o con patología avanzada. En esta situación, es de fundamental importancia realizar una adecuada estratificación del riesgo CV para prescribir la "dosis" adecuada de actividad física que maximice los beneficios y minimice los riesgos.

El presente comentario pretende resumir los aspectos fundamentales de estas directrices novedo$\operatorname{sas}^{(1)}$, que ofrecen recomendaciones concretas en situaciones de patología cardíaca y extracardíaca, destacando la promoción de la actividad física, la evaluación individualizada y la toma de decisiones consensuadas con el paciente.

Identificación de enfermedad cardiovascular y estratificación de riesgo en participantes de deportes recreacionales y competitivos

Altos niveles de actividad física se asocian con reducción de mortalidad por todas las causas, menor riesgo de ECV y menor prevalencia de neoplasias. Sin embargo, como se refirió previamente, el ejerci- cio intenso puede precipitar arritmias ventriculares, siendo la MSC la principal causa de mortalidad relacionada al deporte en atletas. El screening CV preparticipativo tiene como objetivo la detección de desórdenes asociados con MSC, aunque el mejor método permanece en controversia, tanto en atletas $<$ como $\geq 35$ años. Es importante adaptar la estrategia para estas diferentes poblaciones, dado que en $<35$ años la MSC es causada por enfermedades estructurales o eléctricas del corazón (miocardiopatías, canalopatías, anomalías coronarias); mientras que en $\geq 35$ años la enfermedad arterial coronaria (EAC) es la condición primaria determinante de eventos CV mayores adversos (>80\% de las MSC).

La Sociedad Europea de Cardiología define como atleta a "un individuo joven o de edad adulta, tanto amateur o profesional, que practica ejercicio de forma regular y participa en competiciones de deportes oficiales". El atleta recreativo practica deportes por placer y como actividad de tiempo libre, mientras que un atleta competitivo está altamente entrenado, con mayor énfasis en el rendimiento. También se ha clasificado según el mínimo volumen de ejercicio: atletas de élite ( $\geq 10$ horas/semana), competitivos ( $\geq 6$ horas/semana) y recreacionales ( $\geq 4$ horas/semana).

\section{Eventos cardiovasculares mayores adversos relacionados al ejercicio}

Incluyen el paro cardíaco (PC) y la MSC; síndromes coronarios agudos (SCA) como isquemia miocárdica e infarto agudo de miocardio (IAM); ataque isquémico transitorio (AIT) y ataque cerebrovascular (ACV), y taquicardias supraventriculares. El PC se define como un colapso inesperado secundario a causa cardíaca donde la reanimación cardiopulmonar (RCP) o desfibrilación, o ambas, es administra-

Centro Cardiovascular Casa de Galicia. Montevideo, Uruguay.

Correspondencia: Dra. María Victoria Ramos. Correo electrónico: mariavictoriaramos@outlook.es

La autora declara no tener conflictos de intereses.

Recibido Set 9, 2020; aceptado Set 22,2020 
da, independientemente de la sobrevida; la MSC es la muerte súbita e inesperada secundaria a causa cardíaca, o puede atribuirse también en casos de autopsia con corazón estructuralmente normal, sin otra explicación para el evento y una historia consistente con muerte cardíaca relacionada. La asociación temporal con el ejercicio puede ser durante, en la primera hora o luego de la hora y hasta las 24 horas posejercicio.

Los SCA inducidos por ejercicio afectan más frecuentemente a atletas adultos, siendo consecuencia de un accidente de placa y trombosis coronaria en la mayoría de los casos. Sin embargo, en atletas de resistencia, puede producirse por un disbalance oferta-demanda miocárdico de oxígeno, en presencia de placas estables y calcificadas que provocan estenosis coronaria fija. Más de $50 \%$ de los afectados no presenta historia CV previa.

La incidencia de MSC en atletas de competición se estima de 1/1.000.000 - 1/5.000. Esta amplia variación se debe a la variabilidad metodológica de los estudios, con poblaciones heterogéneas. Por otro lado, la denuncia de estos eventos no es obligatoria en muchos países, con la consecuente infraestimación de casos. La evidencia disponible resalta que el sexo masculino, la afrodescendencia, y los jugadores de básquetbol y fútbol, tienen mayor riesgo de MSC.

La etiología de la MSC en atletas jóvenes se atribuye a desórdenes cardíacos estructurales congénitos o genéticos. Sin embargo, las autopsias negativas (referidas como síndrome de muerte súbita arrítmica) alcanzan $44 \%$ en algunos estudios. Por otro lado, la prevalencia de desórdenes cardíacos asociados a MSC en atletas aparentemente sanos es extremadamente baja, de 0,3\% (utilizando herramientas de evaluación no invasiva).

\section{Modalidades de screening de enfermedades cardiovasculares}

El screening CV mediante historia clínica, examen físico o electrocardiograma (ECG) presenta desafíos y limitaciones en atletas < 35 años. Mientras que el ecocardiograma transtorácico (ETT) puede identificar alteraciones estructurales adicionales, no hay evidencia suficiente para recomendarlo de rutina. Por otro lado, en atletas $\geq 35$ años el screening debe enfocarse en la alta prevalencia de EAC. Sin embargo, los estudios para detección de isquemia de rutina en adultos asintomáticos presentan bajo valor predictivo positivo y alto número de falsos positivos. El ECG basal puede detectar desórdenes eléctricos primarios y la estratificación de riesgo permite identificar a individuos con alto riesgo CV que se beneficiarían de estudios adicionales, específicamente una prueba de estrés con ejercicio, también indicada en sintomáticos, para evaluar la respuesta de la presión arterial (PA) al esfuerzo, arritmias inducidas o evaluar el desempeño de adultos que desean comenzar la actividad física.

\section{Actividad física, participación en deportes recreacionales y competitivos}

La actividad física se define como cualquier movimiento corporal producido por el músculo esquelético que resulta en un gasto de energía, mientras que el ejercicio es la actividad física estructurada, repetitiva, con el propósito de mejorar o mantener uno o más componentes de la aptitud física. La aptitud física se expresa en cinco componentes: morfológico (masa corporal en relación con la altura, composición corporal, distribución de la grasa subcutánea, grasa visceral abdominal, densidad ósea y flexibilidad), muscular (fuerza explosiva, fuerza isométrica, resistencia muscular), motor (agilidad, balance, coordinación y velocidad de movimiento), cardiorrespiratorio (capacidad de ejercicio submáxima, poder aeróbico máximo, función cardíaca y pulmonar, PA) y metabólico (tolerancia a la glucosa, sensibilidad a la insulina, metabolismo lipídico y lipoproteico, sustratos de oxidación).

Los principios básicos de la prescripción de ejercicio se han descrito usando el concepto FITT: frecuencia, intensidad, tiempo y tipo. Dentro del tipo, el ejercicio se puede clasificar en aeróbico versus anaeróbico (según el metabolismo), o isotónico versus isométrico (basado en el tipo de contracción muscular). El ejercicio aeróbico se refiere a la actividad realizada a una intensidad que permite que el metabolismo de la energía almacenada se produzca principalmente a través de la glicólisis. Involucra grandes grupos musculares que realizan actividades dinámicas, resultando en incremento sustancial de la frecuencia cardíaca y el gasto energético. Incluye el ciclismo, correr y la natación de intensidad leve a moderada. El ejercicio anaeróbico se refiere al movimiento realizado a alta intensidad, que requiere el metabolismo de la energía almacenada principalmente por glucólisis anaeróbica. Los ejercicios isométricos y de alta intensidad son ejemplos de este último. La mayoría de los deportes tienen tanto componente isométrico como isotónico. Se sugiere una frecuencia mínima de 150 minutos/semana de ejercicio moderado. La intensidad del ejercicio es considerada como el nivel crítico para lograr la aptitud aeróbica y tener impacto más favorable sobre los factores de riesgo. La intensidad absoluta se refiere a la tasa de gasto energético durante el ejercicio y habitualmente se expresa en $\mathrm{kcal} / \mathrm{min}$ o equivalentes metabólicos (METs), mientras que la intensidad relativa se refiere a una fracción de la potencia máxima que se 


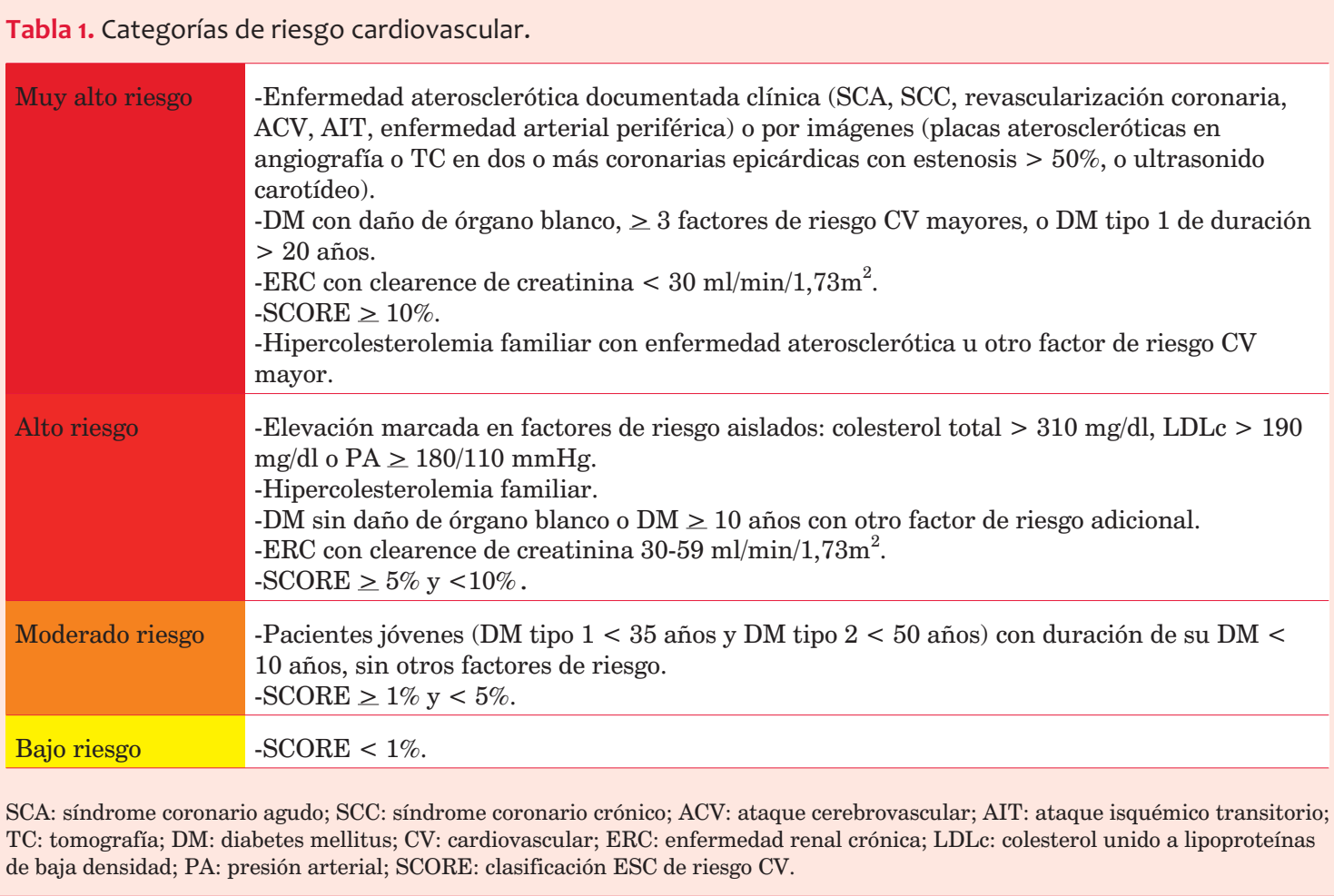

mantiene durante el ejercicio y generalmente se expresa como porcentaje de la capacidad aeróbica máxima $\left(\mathrm{VO}_{2}\right.$ máx) sobre la base de un test de esfuerzo cardiopulmonar. También puede ser expresada como porcentaje de la frecuencia máxima registrada durante una prueba de esfuerzo o predicho utilizando la ecuación: 220 - edad. La intensidad del ejercicio se relaciona inversamente con el tiempo de este.

\section{Recomendaciones de ejercicio en pacientes con factores de riesgo cardiovascular}

Dado que el ejercicio tiene efectos positivos en varios factores de riesgo aterosclerótico independientemente de la edad, el sexo, la etnia o la presencia de comorbilidades, se recomienda que los adultos sanos de todas las edades realicen un mínimo de 150 minutos de entrenamiento de intensidad moderada con ejercicios de resistencia durante cinco días o 75 minutos de ejercicio vigoroso tres veces por semana. Sin embargo, dado que los individuos con múltiples factores de riesgo tienen mayor probabilidad de ECV, es importante calcular la probabilidad subclínica mediante la herramienta SCORE (el riesgo a diez años de eventos fatales se basa en edad, sexo, tabaquismo, PA sistólica y colesterol total) y considerando factores de riesgo individuales como niveles de colesterol total y colesterol unido a lipoproteínas de baja densidad (LDLc) muy altos, diabetes mellitus (DM) o historia familiar de ECV (tabla 1).
En el caso de individuos habitualmente activos y riesgo bajo o moderado, se puede prescribir ejercicio sin ninguna restricción, incluyendo deportes competitivos. Aquellos sedentarios, o con riesgo alto o muy alto, pueden participar de deportes de baja intensidad sin necesidad de evaluaciones adicionales. Sin embargo, si planean practicar ejercicios de alta intensidad (o moderada en casos seleccionados), debería realizarse examen físico, ECG y test de esfuerzo (para identificar EAC importante y presencia de arritmias inducidas por ejercicio). La aparición de síntomas, hallazgos patológicos en el examen físico, ECG o test de esfuerzo, implican la necesidad de evaluación adicional. Aunque un test de esfuerzo normal y buena capacidad funcional se asocian con mejor pronóstico, este estudio tiene sensibilidad limitada en el diagnóstico de EAC obstructiva leve a moderada; sin embargo, no hay recomendación para incorporar la imagen cardíaca de rutina que permita valorar anatomía coronaria, aunque podría considerarse en adultos asintomáticos con riesgo alto o muy alto (DM, historia familiar de EAC) con el objetivo de manejar de forma agresiva los factores de riesgo y realizar tratamiento médico preventivo.

\section{Obesidad}

Se define obesidad cuando el individuo tiene un índice de masa corporal $>30$, o circunferencia de cintura $>94 \mathrm{~cm}$ en hombres y $>80 \mathrm{~cm}$ en mujeres. Se recomienda un mínimo de 150 minutos/semana de 


\begin{tabular}{|c|c|c|c|}
\hline & Diagnóstico & Recomendación & Seguimiento \\
\hline Bajo riesgo & $\begin{array}{l}\text {-Aorta }<40 \mathrm{~mm} \text { (tanto en válvula } \\
\text { aórtica bicúspide como tricúspide). } \\
\text {-Síndrome de Turner sin dilatación } \\
\text { aórtica. }\end{array}$ & $\begin{array}{l}\text { Se permiten todos los } \\
\text { deportes con preferencia } \\
\text { de los de resistencia } \\
\text { sobre los de potencia. }\end{array}$ & Cada dos a tres años. \\
\hline $\begin{array}{l}\text { Riesgo bajo a } \\
\text { intermedio }\end{array}$ & $\begin{array}{l}\text {-Síndrome de Marfan u otra } \\
\text { enfermedad hereditaria de la aorta } \\
\text { torácica sin dilatación aórtica. } \\
\text {-Aorta } 40-45 \mathrm{~mm} \text { (tanto en válvula } \\
\text { aórtica bicúspide como tricúspide). } \\
\text {-Luego de cirugía exitosa de aorta } \\
\text { torácica por válvula bicúspide u otra } \\
\text { situación de bajo riesgo. }\end{array}$ & $\begin{array}{l}\text {-Evitar ejercicios de } \\
\text { intensidad muy alta y } \\
\text { alta, de contacto y } \\
\text { potencia. } \\
\text {-Preferencia de los } \\
\text { deportes de resistencia } \\
\text { sobre los de potencia. }\end{array}$ & Cada uno a dos años. \\
\hline $\begin{array}{l}\text { Riesgo } \\
\text { intermedio }\end{array}$ & $\begin{array}{l}\text {-Dilatación moderada de la aorta ( } 40-45 \\
\text { mm en síndrome de Marfan u otra } \\
\text { enfermedad hereditaria de la aorta } \\
\text { torácica; } 45-50 \mathrm{~mm} \text { (tanto en válvula } \\
\text { aórtica bicúspide como tricúspide), } \\
\text { síndrome de Turner con tamaño aórtico } \\
\text { indexado } 20-25 \mathrm{~mm} / \mathrm{m}^{2} \text {, tetralogía de } \\
\text { Fallot < } 50 \mathrm{~mm} \text { ). } \\
\text {-Luego de cirugía exitosa de aorta } \\
\text { torácica por síndrome de Marfan u otra } \\
\text { enfermedad hereditaria de la aorta } \\
\text { torácica. }\end{array}$ & $\begin{array}{l}\text { Solo deportes de } \\
\text { habilidad, o mixtos, o de } \\
\text { resistencia a baja } \\
\text { intensidad. }\end{array}$ & $\begin{array}{l}\text { Cada seis meses a un } \\
\text { año. }\end{array}$ \\
\hline Alto riesgo & $\begin{array}{l}\text {-Dilatación severa de la aorta (> } 45 \mathrm{~mm} \text { en } \\
\text { síndrome de Marfan u otra enfermedad } \\
\text { hereditaria de la aorta torácica; }>50 \mathrm{~mm} \\
\text { (tanto en válvula aórtica bicúspide como } \\
\text { tricúspide), síndrome de Turner con } \\
\text { tamaño aórtico indexado } \\
>25 \mathrm{~mm} / \mathrm{m}^{2} \text {, tetralogía de Fallot > } 50 \\
\text { mm). } \\
\text {-Luego de cirugía con secuela. }\end{array}$ & $\begin{array}{l}\text { Los deportes están } \\
\text { contraindicados } \\
\text { (temporalmente). }\end{array}$ & $\begin{array}{l}\text { Reevaluación luego de } \\
\text { tratamiento. }\end{array}$ \\
\hline
\end{tabular}

ejercicio de moderada intensidad combinado con tres sesiones semanales de resistencia. La valoración CV preparticipativa debe realizarse en quienes pretendan realizar deportes de alta intensidad, debido a la asociación frecuente con comorbilidades como DM tipo 2, hipertensión arterial (HTA), dislipemia y enfermedad pulmonar.

\section{Hipertensión arterial}

Se considera HTA a la presencia de una PA sistólica $\geq 140 \mathrm{mmHg}$ o PA diastólica $\geq 90 \mathrm{mmHg}$. En quienes la padecen, se recomienda la participación en ejercicios aeróbicos de intensidad moderada a alta al menos de 30 minutos, cinco a siete días a la semana. En el caso de deportes de alta intensidad, debe realizarse la valoración preparticipativa para identificar síntomas inducidos por el ejercicio, respuesta exagerada de la PA o daño de órgano blanco. Las medidas no farmacológicas (restricción en consumo de sal y alcohol, reducción de peso, dieta balanceada y cesación del tabaquismo) son el primer paso de manejo de la HTA. Si estos cambios del estilo de vida no logran el objetivo de PA en tres meses, se recomienda el uso de fármacos antihipertensivos, teniendo en cuenta que los betabloqueantes están prohibidos en ciertas disciplinas de competición, pueden inducir bradicardia y disminuir la capacidad funcional. Los diuréticos están prohibidos en todos los deportes competitivos, siendo de elección los inhibidores del sistema renina-angiotensina-aldosterona y los calcioantagonistas.

\section{Dislipidemia}

La actividad física tiene efectos favorables en el metabolismo lipídico: reducción de triglicéridos en $50 \%$ y aumento de colesterol unido a lipoproteínas de alta densidad (HDLc) de 5\% a 10\%. Se recomiendan ejercicios de alta intensidad en caso de hipertrigliceridemia o hipercolesterolemia, previa evaluación CV preparticipativa que incluya: síntomas, test de esfuerzo, test de imagen o anatomía coronaria mediante tomografía (particularmente en casos de 
hipercolesterolemia familiar). Las estatinas son superiores al ejercicio y modificaciones en el estilo de vida en reducir el LDLc, aunque se sugiere fuertemente su asociación.

\section{Diabetes mellitus}

El riesgo de desarrollar DM tipo 2 es 50\%-80\% mayor en individuos físicamente inactivos en comparación con los activos. El ejercicio mejora el control glicémico, reduce la grasa visceral y la resistencia a la insulina. Estudios observacionales han mostrado menor mortalidad en DM tipo 1 y 2 con la práctica de ejercicio. Tanto el entrenamiento aeróbico como de resistencia son efectivos para el control de la glicemia, reducción de la PA, pérdida de peso, capacidad de ejercicio pico y dislipemia. Se recomienda una práctica de moderada intensidad de 30 minutos diariamente con entrenamiento de resistencia de 15 minutos. Todos los individuos con DM deben someterse a una evaluación CV preparticipativa antes de ingresar a un programa de alta intensidad, debido a la alta probabilidad de EAC subclínica.

Ejercicio y deportes en el individuo añoso Se definen como aquellos $>65$ años. Similar a lo que ocurre con la población general, la mayor capacidad de ejercicio se asocia con reducción en la mortalidad, menor riesgo de ECV y enfermedad metabólica, mejor control de los factores de riesgo CV, función cognitiva preservada, mantenimiento de la competencia muscular, coordinación y balance (disminuyendo el riesgo de caídas). El ejercicio de moderada intensidad es generalmente seguro en individuos sanos. Alcanzar 150 minutos/semana de ejercicio aeróbico de moderada intensidad se asocia con descensos de $30 \%$ en morbimortalidad, discapacidad, fragilidad y demencia, comparado con individuos inactivos. La evaluación anual con test de esfuerzo se recomienda en atletas que practican deportes de alto nivel.

\section{Ejercicio en escenarios clínicos}

\section{Programas de ejercicio recreacional y competitivo en enfermedad arterial coronaria}

Los ejercicios de intensidad moderada a vigorosa se asocian a reducción en la incidencia de eventos adversos $\mathrm{CV}$, pero cuando son prolongados y de alta intensidad, se han vinculado a incremento en el calcio coronario (marcador de aterosclerosis) sin cambios en la mortalidad. La evaluación clínica de individuos asintomáticos con probable síndrome coronario crónico incluye: evaluación del riesgo de ECV, considerar la intensidad del ejercicio que pretenden realizar, evaluación clínica (incluyendo test de esfuerzo) y diagnósticos adicionales en casos seleccionados. Dado que la detección de EAC por imagen no provee información de la reserva de flujo coronario, debe realizarse un estudio funcional que permita detectar la presencia de isquemia miocárdica inducida (incluyendo el test de esfuerzo máximo, aun cuando tenga menor especificidad que otras técnicas). Existen tres resultados posibles: en caso de evaluación clínica normal, la presencia de EAC relevante se asume improbable; en caso de resultados dudosos o no interpretables, se recurre a un estudio funcional más específico que incluya evaluación por imágenes, y en caso de test positivo se realizará una cineangiocoronariografía (CACG).

Los individuos en riesgo de EAC o asintomáticos con EAC detectada por screening deben recibir tratamiento agresivo de los factores de riesgo aterosclerótico. Sólo deberían restringirse los deportes competitivos cuando exista riesgo sustancial de eventos adversos por estudio funcional, o evidencia de progresión de la enfermedad.

Los individuos con síndrome coronario crónico establecido (angina estable, asintomática o sintomática $<1$ año luego de SCA, revascularización reciente y asintomáticos o sintomáticos $>1$ año después del diagnóstico inicial o revascularización) que no muestran anomalías en el test de esfuerzo o estudio funcional con imágenes, o tienen función ventricular izquierda preservada (FEVI > 50\%) y sin arritmias inducidas, se consideran de bajo riesgo para eventos adversos inducidos por ejercicio. Se aplican algunas restricciones en caso de deportes de alta intensidad, mixtos y de resistencia para pacientes $>60$ años. Si existe isquemia inducible durante el estudio funcional pese a tratamiento adecuado, deberá realizarse una CACG y el retorno a la actividad deportiva será gradual en 3-6 meses luego de la revascularización exitosa. Cuando la isquemia no responde al tratamiento adecuado, se deben restringir los deportes competitivos, con excepción de los de baja intensidad.

Luego de un SCA, revascularización mediante cirugía o intervención coronaria percutánea, los pacientes deben referirse a un programa de rehabilitación cardíaca basado en ejercicio precoz, por 8-12 semanas luego del evento. Todos los tipos de actividad deportiva pueden considerarse a una intensidad apropiada, prestando especial atención al desarrollo de síntomas. En atletas de competición, se requiere ETT, test de esfuerzo máximo o test cardiopulmonar para estratificar el riesgo antes de retornar a la actividad.

El origen anómalo de las arterias coronarias (izquierda y derecha) afecta a $0,44 \%$ de la población 
general adolescente. Sin embargo, se considera una causa frecuente de MSC en atletas jóvenes. Ángor, síncope durante el ejercicio y MSC pueden ser las formas de manifestación, aunque dos tercios permanecen asintomáticos. Episodios reiterados de isquemia y posterior fibrosis (sustrato para arritmias ventriculares durante el ejercicio) serían los implicados en el mecanismo de MSC. La isquemia se produce por compresión del vaso anómalo en su trayecto entre la aorta y la arteria pulmonar, ángulo o curso intramural del mismo. La elección del deporte competitivo que puede practicarse depende del tipo de anomalía y de si existe isquemia inducible (evidenciada mediante test de esfuerzo). Las anomalías que presentan un ángulo agudo de origen desde la aorta o trayecto entre la aorta y la arteria pulmonar se consideran de alto riesgo para PC/MSC y se sugiere la corrección mediante cirugía.

El puente miocárdico se refiere a la condición en que el miocardio recubre un segmento de una arteria coronaria epicárdica, más frecuentemente la descendente anterior izquierda. La prevalencia es variable (desde $0,5 \%-12 \%$ a $5 \%-75 \%$ ). Tradicionalmente se ha considerado benigno, sin embargo, reviste especial interés su asociación con isquemia durante el ejercicio (manifestada por ángor o síncope). La evaluación de los individuos afectados implica su reconocimiento anatómico (número de puentes, profundidad y trayecto) y evaluar la presencia de isquemia, que condicionará una eventual restricción de participar en deportes competitivos.

\section{Recomendaciones de ejercicio en individuos con insuficiencia cardíaca crónica}

Los programas de entrenamiento en pacientes estables (bajo tratamiento médico óptimo) se consideran seguros y altamente recomendables, dada la mejor tolerancia al ejercicio, calidad de vida y modesto efecto en mortalidad por todas las causas y hospitalización por insuficiencia cardíaca (IC). Previo al inicio de la actividad física, debe asegurarse la estabilidad clínica, evaluar las comorbilidades y severidad de la IC (péptidos natriuréticos y ETT) y la capacidad funcional mediante test de esfuerzo máximo. Es fundamental optimizar el tratamiento, incluyendo el implante de dispositivos, de ser necesario. Los pacientes asintomáticos con fracción de eyección del ventrículo izquierdo (FEVI) preservada $(\geq 50 \%)$ o en rango intermedio (40\%-49\%) óptimamente tratados, podrían participar en algunos deportes competitivos, en ausencia de arritmias o hipotensión inducida por ejercicio. Los pacientes asintomáticos, pero con FEVI reducida y óptimamente tratados, sólo pueden practicar formas específicas de deportes competitivos de baja intensidad.
Los mismos criterios se aplican para deportes recreacionales. Los pacientes receptores de trasplante cardíaco que participan en programas de rehabilitación basada en ejercicio revelan resultados favorables en cuanto a reingreso hospitalario y sobrevida a largo plazo. La reinervación contribuye a mejorar la capacidad funcional en el primer año, permitiendo realizar actividades de alta intensidad (dado que la mayor limitación del ejercicio de resistencia es la respuesta cronotrópica reducida).

\section{Recomendaciones de ejercicio en individuos con valvulopatías}

Las valvulopatías afectan 1\%-2\% de individuos jóvenes en la población general. No existen estudios prospectivos que evalúen el impacto del ejercicio en la progresión de la enfermedad (aunque se ha postulado que el volumen sistólico aumentado, contracciones mecánicas vigorosas y el cronotropismo positivo puedan acelerarla). El manejo general requiere una evaluación del estado sintomático, capacidad funcional, naturaleza de la lesión valvular y el impacto de las condiciones de carga en la estructura y función cardíacas. A todos los individuos se les realizará historia clínica, examen físico, ECG, ETT y test de esfuerzo. Aquellos asintomáticos con disfunción valvular leve a moderada (en el caso de las lesiones regurgitantes), función ventricular izquierda preservada, buena capacidad funcional sin isquemia inducible en el test de esfuerzo, sin respuesta hemodinámica anormal ni arritmias, se consideran de bajo riesgo y, por ende, pueden participar de todos los deportes. Quienes no cumplan con estos requisitos, se consideran de alto riesgo y requieren evaluación invasiva. Específicamente se destaca el prolapso de válvula mitral, dado que, si bien el pronóstico general es excelente, la presencia de inversión de ondas $\mathrm{T}$ en derivaciones inferiores y extrasistolía ventricular con morfología sugestiva de origen en el VI, debería ser valorada con resonancia nuclear magnética (RNM) cardíaca para descartar la presencia de fibrosis miocárdica (marcador de riesgo de MSC).

\section{Recomendaciones de ejercicio en individuos con aortopatías}

Edad avanzada, sexo masculino, HTA de larga data y presencia de aneurisma aórtico son factores de riesgo para presentar disección de aorta. Sin embargo, los desórdenes genéticos del tejido conectivo (síndrome de Marfan, Loeys Dietz, Turner o Ehlers Danlos) y válvula aórtica bicúspide tienen riesgo a edades más tempranas. También la historia familiar de disección aórtica o MSC y diámetros de aorta mayores, implican alto riesgo. Dado el incremento 
de la PA y del estrés parietal asociado a deportes de alta intensidad, podría existir riesgo de dilatación de la aorta y disección. Sin embargo, la actividad física regular se recomienda en pacientes con patología, aun cuando la aorta esté dilatada (dentro de determinados rangos, ver tabla 2), dado que no existen estudios randomizados de este tópico particular, que demuestren efectos adversos. Previamente a la prescripción de ejercicio, se realizará estratificación de riesgo en base a imagen de la aorta (tomografía computada [TC] o RNM y un test de esfuerzo para valorar la respuesta de la PA).

\section{Recomendaciones de ejercicio en individuos con miocardiopatías, miocarditis y pericarditis}

Miocardiopatía hipertrófica (MCH): se diagnostica por hipertrofia ventricular izquierda (HVI) inexplicada en cualquier segmento $\geq 15 \mathrm{~mm}$ de grosor de fin de diástole (por ETT, TC o RNM) o $\geq 13 \mathrm{~mm}$ en caso de historia familiar o test genético positivo. El ejercicio incrementa los riesgos de PC/MSC en estos individuos, aunque estudios recientes indican que este riesgo es menor de lo que se consideraba inicialmente. Por tanto, la restricción sistemática de los deportes competitivos en todos los individuos probablemente esté injustificada, siendo necesaria una valoración cuidadosa mediante historia personal (edad del paciente y años de ejercicio previo al diagnóstico) y familiar, severidad del fenotipo de $\mathrm{MCH}$ y presencia de factores de riesgo convencionales para PC/MSC. Se recomienda evaluar el estado sintomático, arritmias -taquicardia ventricular no sostenida- (mediante Holter, que presenta mayor rendimiento frente al ECG), ETT (espesor parietal, gradiente a nivel del tracto de salida del VI, diámetro auricular izquierdo), RNM (la presencia de realce tardío con gadolinio $\geq 15 \%$ del miocardio del VI se asocia con riesgo incrementado de arritmias ventriculares y MSC), test de esfuerzo (descenso de la PA $>20 \mathrm{mmHg}$ o hipotensión, síntomas y arritmias inducidas por el ejercicio son marcadores de riesgo) y el uso de score de riesgo. Es importante considerar que la edad del individuo tiene impacto en el riesgo, ya que, en uno de los estudios más grandes, $65 \%$ de las MSC se produjeron en $\leq 17$ años. En el caso de portadores de la mutación que no expresan el fenotipo, no debería restringirse el deporte, siempre que se mantenga un seguimiento periódico.

Miocardiopatía arritmogénica: se define por la presencia de reemplazo fibro-adiposo principalmente de los ventrículos, con riesgo de MSC por arritmias ventriculares. El diagnóstico se basa en criterios probabilísticos que incluyen alteraciones electrofisiológicas, anatómicas, funcionales y clínicas. Dado que las recomendaciones se basan en es- tudios de la influencia del ejercicio en la progresión de la enfermedad (y riesgo de MSC) en cohortes con la forma clásica (afectación predominante del ventrículo derecho), pueden no ser extrapolables a los casos con afectación del VI. Existe una proporción significativa de atletas jóvenes con MSC que padecen esta patología. Los factores de riesgo establecidos para implante de cardiodesfibrilador (CDI) son MSC, síncope, taquicardia ventricular y deterioro de la función ventricular derecha o izquierda. Los programas de entrenamiento regular y de alta intensidad se asocian con progresión de la enfermedad y peor pronóstico. La valoración incluye: historia clínica (presencia de síntomas, específicamente síncope), ECG basal (ondas T invertidas $\geq 3$ derivaciones precordiales o $\geq 2$ derivaciones inferiores confieren riesgo adicional de PC/MSC). El Holter es importante en la detección de arritmias, ya que la presencia de taquicardia ventricular no sostenida (TVNS) o alta carga de ectopía ventricular $(\geq$ 1.000/24 horas) aumenta el riesgo de arritmias fatales. Debe valorarse el grado de compromiso ventricular mediante ETT o RNM (mayor sensibilidad en la evaluación de infiltración y cicatriz). El test de esfuerzo provee información de la capacidad funcional y estratificación de riesgo. La recomendación general en estos pacientes es desalentar la participación en deportes de alta intensidad porque se asocian con progresión acelerada de la enfermedad, mayor riesgo de arritmias ventriculares y eventos mayores adversos (aplicable también en caso de genotipo positivo sin manifestación fenotípica).

Miocardiopatía no compactada: se caracteriza por trabeculación ventricular izquierda prominente y recesos profundos que comunican con la cavidad. La presentación clínica incluye disfunción sistólica progresiva, taquiarritmias ventriculares y eventos tromboembólicos. Es importante destacar que $8 \%$ de los atletas presentan hipertrabeculación, pero la sospecha de miocardiopatía sólo debería considerarse cuando asocia FEVI $<50 \%$, síntomas compatibles o antecedentes familiares. El riesgo se basa en la presencia de síntomas, arritmias ventriculares y severidad de la disfunción ventricular, en estos casos, no se recomiendan los deportes de alta intensidad.

Miocardiopatía dilatada: se caracteriza por disfunción ventricular izquierda o biventricular no explicada por condiciones de carga anormales o EAC. Tanto la etiología como el espectro clínico son variables. El riesgo de MSC alcanza 2\%-3\% anual y se incrementa a medida que la FEVI desciende y la capacidad funcional disminuye. La dilatación del VI en individuos entrenados sin evidencia de disfunción sistólica o historia familiar, representa una adapta- 
ción fisiológica. Los pacientes asintomáticos con leve disfunción ventricular (FEVI 45\%-50\%), sin arritmias inducidas durante el ejercicio, ni fibrosis miocárdica significativa, pueden participar en la mayoría de los deportes (con excepción de variantes patogénicas específicas, como la mutación en lamin $\mathrm{A} / \mathrm{C})$.

Miocarditis: inflamación no isquémica del miocardio, que puede causar disfunción sistólica y arritmias. La infección viral es la etiología más frecuente en países desarrollados. La presentación clínica es altamente variable: desde síntomas inespecíficos a arritmias ventriculares, IC, shock cardiogénico o MSC. Aproximadamente, $50 \%$ de los individuos presentan recuperación completa de la FEVI al mes, 25\% muestra persistencia de la disfunción y 12\%-25\% progresa a miocarditis fulminante. La RNM tiene excelente sensibilidad para detectar hiperemia miocárdica, inflamación, edema y cicatriz focal. Series de casos publicadas han establecido que la miocarditis es factor de riesgo de MSC, explicando el 2\%-20\% de los episodios en atletas, por tal motivo, se recomienda la abstención de practicar deportes mientras exista inflamación activa presente, independientemente de la edad, sexo o severidad de la disfunción ventricular. Las sociedades científicas europeas y americanas recomiendan a estos pacientes abstenerse de practicar ejercicio de moderada a alta intensidad por un período de tres a seis meses. Luego de la recuperación, debe evaluarse el riesgo de $\mathrm{MSC}$ asociado a ejercicio mediante estudios de imagen, prueba de esfuerzo y Holter. El retorno a la actividad deportiva puede considerarse en individuos asintomáticos, con marcadores inflamatorios y troponinas dentro de rango normal, FEVI conservada, ausencia de inflamación en curso o fibrosis miocárdica en la RNM, buena capacidad funcional y ausencia de arritmias complejas durante el ejercicio o monitoreo continuo. La excepción son los pacientes con cicatriz miocárdica extensa (> $20 \%$ de realce tardío con gadolinio en RNM).

Pericarditis: inflamación del pericardio, habitualmente de etiología viral. Aunque el pronóstico es excelente, debe evitarse realizar actividad física durante la fase activa, retomando la misma una vez resuelto el episodio, habitualmente al mes en casos leves, que puede prolongarse a tres meses cuando la afección es severa.

\section{Recomendaciones de ejercicio en individuos con arritmias y canalopatías}

Conceptualmente, todas las adaptaciones cardíacas funcionales y estructurales del ejercicio intenso regular pueden contribuir al desarrollo de arritmias, tanto auriculares, nodales, como ventriculares.
Fibrilación auricular (FA): aunque la actividad física ha demostrado prevenir la aparición de FA a través de la modificación de los factores de riesgo, los atletas de resistencia de alta intensidad presentan más frecuentemente FA, sugiriendo la existencia de una curva en U relacionada al nivel de ejercicio. Antes de realizar una recomendación sobre el inicio de deportes en pacientes portadores de FA, debe evaluarse la presencia de enfermedad estructural cardíaca subyacente y preexcitación. También es importante excluir otras causas como hipertiroidismo, abuso de alcohol u otras drogas. El adecuado control de la frecuencia ventricular durante el ejercicio puede ser difícil de alcanzar, con la consecuente aparición de síntomas limitantes, siendo necesaria la combinación de fármacos con efecto cronotrópico negativo. El control del ritmo igualmente es complejo: los fármacos clase III no se recomiendan en pacientes jóvenes por sus efectos secundarios, mientras que los de clase I deben asociarse a otros antiarrítmicos para evitar la aparición de flutter con conducción 1:1. Cuando estas estrategias fallan o no es deseable el uso de fármacos, se puede considerar la ablación.

Taquicardia supraventricular (TSV) y síndrome de Wolf-Parkinson-White: la prevalencia de preexcitación en la población general es de 0,1\%-0,3\%. Las TSV sin preexcitación ni cardiopatía estructural no ponen en riesgo la vida, aunque pueden ser sintomáticas durante el ejercicio. El síncope es poco frecuente. Los atletas con TSV deben detener el ejercicio cuando perciban síntomas, y realizar maniobras vagales con el objetivo de interrumpir la taquicardia, pudiendo retomar la actividad posteriormente. En caso de recurrencias frecuentes se sugiere la realización de ablación. La preexcitación puede asociarse con MSC $(0,15 \%-0,20 \%$ por la aparición de FA que conduce a través de la vía accesoria, degenerando en fibrilación ventricular), por tal motivo, debe excluirse la presencia de vías accesorias de conducción oculta (mediante adenosina o maniobras vagales). Debe descartarse la presencia de cardiopatía estructural (MCH o anomalía de Ebstein). La preexcitación intermitente usualmente indica que las propiedades de la vía accesoria son de bajo riesgo, aunque el estímulo adrenérgico puede potenciarlas. La ablación se recomienda en atletas competitivos y recreacionales con preexcitación y arritmias documentadas. En los casos asintomáticos, debe evaluarse el riesgo de MSC mediante estudio electrofisiológico (EEF): en caso de alto riesgo (FA o TSV mediada por vía accesoria inducible en EEF, $\mathrm{R}$-R en FA $\leq 250 \mathrm{~ms}$, período refractario anterógrado $\leq 250 \mathrm{~ms}$, múltiples vías, localización septal), también se procederá a la ablación. Luego del proce- 
Tabla 3. Principales recomendaciones de la guía.

\begin{tabular}{|c|c|c|c|}
\hline & Recomendaciones & Clase & Nivel \\
\hline $\begin{array}{l}\text { Recomendaciones } \\
\text { generales }\end{array}$ & $\begin{array}{l}150 \mathrm{minutos} / \text { semana de ejercicio aeróbico de moderada intensidad o } \\
75 \mathrm{minutos} / \mathrm{semana} \text { (de vigorosa intensidad) en todos los adultos sanos. }\end{array}$ & I & A \\
\hline \multirow{3}{*}{$\begin{array}{l}\text { Evaluación CV en } \\
\text { individuos sanos }> \\
35 \text { años }\end{array}$} & $\begin{array}{l}\text { Individuos con riesgo de ECV bajo a moderado pueden participar en todos } \\
\text { los deportes recreativos sin evaluación adicional. }\end{array}$ & IIa & $\mathrm{C}$ \\
\hline & $\begin{array}{l}\text { En atletas de competición debe considerarse realizar screening con } \\
\text { historia familiar, evaluación de síntomas, examen físico y ECG. }\end{array}$ & IIa & $\mathrm{C}$ \\
\hline & $\begin{array}{l}\text { Se realizará prueba de esfuerzo en sedentarios con riesgo } \mathrm{CV} \text { alto o muy } \\
\text { alto que deseen realizar ejercicio intenso. }\end{array}$ & IIa & $\mathrm{C}$ \\
\hline $\begin{array}{l}\text { Consideraciones } \\
\text { especiales en } \\
\text { pacientes con } \\
\text { FRCV }\end{array}$ & $\begin{array}{l}\text { Entrenamiento de resistencia } \geq 3 \text { veces/semana junto a actividad aeróbica } \\
\text { de moderada a vigorosa intensidad (al menos } 30 \text { minutos, } 5-7 \\
\text { días/semana) para reducir el riesgo } \mathrm{CV} \text {. }\end{array}$ & I & $\mathrm{A}$ \\
\hline Individuos con SCC & $\begin{array}{l}\text { Estratificación de riesgo de eventos adversos inducidos por ejercicio previo } \\
\text { a su inicio. }\end{array}$ & I & $\mathrm{C}$ \\
\hline $\begin{array}{l}\text { Retorno al ejercicio } \\
\text { luego de SCA }\end{array}$ & $\begin{array}{l}\text { Rehabilitación cardíaca basada en ejercicio en todos los individuos con } \\
\text { EAC para reducir la mortalidad CV y rehospitalización. }\end{array}$ & I & $\mathrm{A}$ \\
\hline \multirow[t]{2}{*}{$\begin{array}{l}\text { Individuos } \\
\text { portadores de IC }\end{array}$} & $\begin{array}{l}\text { En IC con FEVI de rango intermedio, se recomienda rehabilitación } \\
\text { cardíaca basada en ejercicio en todos los pacientes estables para mejorar } \\
\text { la capacidad funcional, calidad de vida y reducir la frecuencia de } \\
\text { rehospitalización. }\end{array}$ & I & A \\
\hline & $\begin{array}{l}\text { Antes de iniciar la actividad deportiva, debe optimizarse el tratamiento, } \\
\text { incluyendo el implante de dispositivos si corresponde. }\end{array}$ & I & $\mathrm{C}$ \\
\hline \multirow[t]{7}{*}{ Valvulopatías } & $\begin{array}{l}\text { Se puede participar de todas las disciplinas deportivas en caso de estenosis } \\
\text { o insuficiencia aórtica leve e insuficiencia mitral leve. }\end{array}$ & I & $\mathrm{C}$ \\
\hline & $\begin{array}{l}\text { Deportes de intensidad leve a moderada puede considerarse en estenosis } \\
\text { aórtica moderada con FEVI } \geq 50 \% \text {, buena capacidad funcional y } \\
\text { respuesta normal de la PA en prueba de esfuerzo. }\end{array}$ & $\mathrm{IIb}$ & $\mathrm{C}$ \\
\hline & $\begin{array}{l}\text { Pueden participar de todos los deportes los individuos asintomáticos con } \\
\text { insuficiencia aórtica moderada, VI no dilatado, FEVI > 50\% y prueba de } \\
\text { esfuerzo normal. }\end{array}$ & IIa & $\mathrm{C}$ \\
\hline & $\begin{array}{l}\text { No se recomienda la participación en disciplinas de moderada o alta } \\
\text { intensidad en individuos con insuficiencia aórtica severa y/o FEVI }=50 \% \\
\text { y/o arritmias inducidas por ejercicio. }\end{array}$ & III & $\mathrm{C}$ \\
\hline & $\begin{array}{l}\text { En individuos con insuficiencia mitral moderada }{ }^{(1)} \text { a severa }^{(2)} \text {, se puede } \\
\text { participar de todos los deportes sí: } \\
\text { - DDFVI }<60 \mathrm{~mm} \\
\text { - FEVI } \geq 60 \% \\
\text { - PAP reposo }<50 \mathrm{mmHg} \\
\text { - Prueba de esfuerzo normal. }\end{array}$ & $\begin{array}{cc}1 & 2 \\
\mathrm{IIa} & \mathrm{IIb}\end{array}$ & \\
\hline & $\begin{array}{l}\text { En estenosis mitral leve se recomienda participación en todos los deportes } \\
\text { con PAP reposo }<40 \mathrm{mmHg} \text { y prueba de esfuerzo normal. }\end{array}$ & I & $\mathrm{C}$ \\
\hline & $\begin{array}{l}\text { En estenosis mitral moderada se recomiendan deportes de leve a } \\
\text { moderada intensidad con PAP reposo }<40 \mathrm{mmHg} \text { y prueba de esfuerzo } \\
\text { normal. }\end{array}$ & $\mathrm{IIb}$ & $\mathrm{C}$ \\
\hline
\end{tabular}


(viene de la página anterior)

\begin{tabular}{|c|c|c|c|}
\hline & Recomendaciones & Clase & Nivel \\
\hline \multirow[t]{4}{*}{ Miocardiopatías } & $\begin{array}{l}\text { La participación en ejercicio de alta intensidad no se recomienda en } \\
\text { individuos con } \mathrm{MCH} \text { con marcadores de riesgo (síntomas, PC, síncope } \\
\text { inexplicado, riesgo ESC } \geq 4 \% \text { a cinco años, gradiente del TSVI en reposo } \\
>30 \mathrm{mmHg} \text {, respuesta anormal de PA en ejercicio, arritmias inducidas en } \\
\text { ejercicio). }\end{array}$ & III & $\mathrm{C}$ \\
\hline & $\begin{array}{l}\text { En miocardiopatía arritmogénica la participación deportiva de baja a } \\
\text { moderada intensidad se recomienda en pacientes sin historia de PC o } \\
\text { arritmias ventriculares, síncope inexplicado, anomalías estructurales, < } \\
500 \mathrm{EV} / 24 \text { horas y sin evidencia de arritmias inducidas en ejercicio. }\end{array}$ & $\mathrm{IIb}$ & $\mathrm{C}$ \\
\hline & $\begin{array}{l}\text { Los pacientes con VI no compactado, no deberían participar en deportes } \\
\text { de alta intensidad si presentan: síntomas, FEVI < } 40 \% \text { o arritmias } \\
\text { ventriculares complejas en Holter o prueba de esfuerzo. }\end{array}$ & III & $\mathrm{C}$ \\
\hline & $\begin{array}{l}\text { Puede considerarse la participación en actividad de alta intensidad en } \\
\text { pacientes con MCD asintomáticos con: FEVI } 45 \%-50 \% \text {, ausencia de } \\
\text { arritmias ventriculares complejas en Holter o prueba de esfuerzo, } \\
\text { ausencia de realce tardío en RNM, incremento de la FEVI } 10 \%-15 \% \\
\text { durante el ejercicio y ausencia de genotipo de alto riesgo (lamin A/C o } \\
\text { filamin C). }\end{array}$ & $\mathrm{IIb}$ & $\mathrm{C}$ \\
\hline Miocarditis & $\begin{array}{l}\text { El retorno a todas las formas de ejercicio debe considerarse luego de 3-6 } \\
\text { meses en pacientes asintomáticos, con troponinas y marcadores } \\
\text { inflamatorios normales, FEVI normal, sin evidencia de inflamación en } \\
\text { curso o fibrosis miocárdica en RNM, buena capacidad funcional y ausencia } \\
\text { de arritmias ventriculares complejas en Holter o ejercicio. }\end{array}$ & IIa & $\mathrm{C}$ \\
\hline Pericarditis & $\begin{array}{l}\text { El retorno a todas las formas de ejercicio se recomienda luego de } 30 \text { días a } \\
3 \text { meses para aquellos individuos completamente recuperados, } \\
\text { dependiendo de la severidad del episodio. }\end{array}$ & I & $\mathrm{C}$ \\
\hline \multirow[t]{7}{*}{$\begin{array}{l}\text { Arritmias y } \\
\text { canalopatías }\end{array}$} & $\begin{array}{l}\text { La frecuencia ventricular en FA alcanzada durante el ejercicio debe } \\
\text { considerarse en cada individuo (mediante evaluación de síntomas o } \\
\text { monitoreo electrocardiográfico) para alcanzar un adecuado control. }\end{array}$ & IIa & $\mathrm{C}$ \\
\hline & $\begin{array}{l}\text { No se recomiendan los deportes de contacto o con riesgo de trauma en } \\
\text { aquellos pacientes que requieran terapia anticoagulante. }\end{array}$ & III & A \\
\hline & $\begin{array}{l}\text { La ablación de vía accesoria se recomienda en atletas con preexcitación y } \\
\text { arritmias documentadas. }\end{array}$ & I & $\mathrm{C}$ \\
\hline & $\begin{array}{l}\text { En deportistas con } \geq 2 \mathrm{EV} \text { en ECG basal ( } \mathrm{o} \geq 1 \text { en atletas de alta } \\
\text { resistencia) se recomienda una evaluación detallada (incluyendo historia } \\
\text { familiar) para descartar patología estructural o arrítmica. }\end{array}$ & I & $\mathrm{C}$ \\
\hline & $\begin{array}{l}\text { La participación en deportes de alta intensidad no se recomienda en } \\
\text { individuos con QT }>500 \mathrm{~ms} \text { o síndrome de QT largo genéticamente } \\
\text { confirmado con QTc } \geq 470 \mathrm{~ms} \text { en hombres y } \geq 480 \mathrm{~ms} \text { en mujeres, aún } \\
\text { bajo tratamiento betabloqueante. }\end{array}$ & III & B \\
\hline & $\begin{array}{l}\text { Luego del implante de cardiodesfibrilador en pacientes con síndrome de } \\
\text { Brugada, puede considerarse el retorno a la actividad deportiva en } \\
\text { aquellos que no hayan experimentado arritmias durante tres meses. }\end{array}$ & IIa & $\mathrm{C}$ \\
\hline & $\begin{array}{l}\text { La participación en deportes y ejercicio (excepto de colisión) debe } \\
\text { considerarse en individuos portadores de MPD sin sustrato patológico } \\
\text { para arritmias fatales. }\end{array}$ & IIa & $\mathrm{C}$ \\
\hline $\begin{array}{l}\text { Cardiopatías } \\
\text { congénitas }\end{array}$ & $\begin{array}{l}\text { Evaluar la función ventricular, presión arterial pulmonar, tamaño aórtico } \\
\text { y riesgo arrítmico, se recomienda en todos los atletas. }\end{array}$ & I & $\mathrm{C}$ \\
\hline \multicolumn{4}{|c|}{$\begin{array}{l}\text { CV: cardiovascular; ECV: enfermedad cardiovascular; ECG: electrocardiograma; FRCV: factores de riesgo cardiovascular; SCC: sín- } \\
\text { drome coronario crónico; SCA: síndrome coronario agudo; EAC: enfermedad arterial coronaria; IC: insuficiencia cardíaca; FEVI: } \\
\text { fracción de eyección del ventrículo izquierdo; PA: presión arterial; DDFVI: diámetro diastólico final del ventrículo izquierdo; PAP: } \\
\text { presión arterial pulmonar; MCH: miocardiopatía hipertrófica; ESC: Sociedad Europea de Cardiología (sigla en inglés); TSVI: tracto } \\
\text { de salida del ventrículo izquierdo; PC: parada cardíaca; EV: extrasistolía ventricular; RNM: resonancia nuclear magnética; FA: fi- } \\
\text { brilación auricular; Qtc: QT corregido; MPD: marcapasos definitivo. }\end{array}$} \\
\hline
\end{tabular}


dimiento, puede retomarse la actividad deportiva en uno a tres meses.

\section{Extrasistolía ventricular y taquicardia ventricular no sostenida}

La extrasistolía ventricular (EV) puede ser marcadora de patología cardíaca subyacente, con implicancia pronóstica aun en pacientes asintomáticos. Aunque no existe punto de corte absoluto, se considera que $>2.000 \mathrm{EV}$ en 24 horas se asocian con $30 \%$ de posibilidad de que exista una cardiopatía subyacente. Para estratificar el riesgo de las EV, es importante evaluar-además de la frecuencia-, la morfología y respuesta al ejercicio (su reducción o resolución con carga incremental es típica de arritmias benignas). En aquellos individuos que deseen practicar deportes debe descartarse la presencia de cardiopatía estructural o condiciones arritmogénicas familiares mediante los estudios que previamente se han mencionado.

Síndrome de QT largo: en los casos de síndrome adquirido, la actividad deportiva queda prohibida hasta corregir la causa desencadenante. En los casos de síndrome congénito, el riesgo de eventos durante el ejercicio depende de la mutación, siendo mayor en el síndrome QT largo tipo 1. Los pacientes sintomáticos no deben participar de deportes competitivos, y en el caso del tipo 1, no deben realizar inmersión en agua fría.

Síndrome de Brugada: no existen reportes que asocien al ejercicio con eventos cardíacos, por ende, los pacientes asintomáticos con patrón tipo I espontáneo pueden competir en todos los deportes, excepto en aquellos con incremento de la temperatura corporal $>39^{\circ} \mathrm{C}$. En los portadores de CDI, puede retomarse la actividad luego de $\geq 3$ meses asintomáticos.

Implante de dispositivos: las recomendaciones son más liberales en portadores de marcapasos (MP) que en los que poseen CDI. Los pacientes con MP pueden participar en deportes competitivos o recreativos en ausencia de cardiopatía significativa que los prohíba; sin embargo, deben evitarse en las primeras semanas posteriores al implante por el riesgo de dislocación del electrodo. Tampoco se recomiendan las actividades asociadas a riesgo de trauma en tórax. En el caso de los CDI, deben consi- derarse aspectos como el efecto del ejercicio en la progresión de la cardiopatía de base, el aumento de choques apropiados e inapropiados en deportes competitivos versus recreacionales, y la adecuada programación del dispositivo.

\section{Recomendaciones de ejercicio en individuos con cardiopatías congénitas}

El asesoramiento individualizado de los portadores de cardiopatía congénita (CC) es fundamental. Dado que existe considerable variación de las consecuencias hemodinámicas según el tipo de cardiopatía, debe valorarse el riesgo mediante historia clínica, examen físico, ECG, ETT y test de esfuerzo (idealmente test cardiopulmonar). La MSC en portadores de CC es rara ( $<0,1 \%$ anual), por ende, el ejercicio moderado a vigoroso se considera seguro en la mayoría de estos pacientes, incluso si se encuentran sintomáticos (clase funcional II-III).

\section{Conclusiones}

Esta guía es la primera en su tipo publicada por la Sociedad Europea de Cardiología. Aunque en la mayoría de las recomendaciones, el nivel de evidencia es bajo, destaca la flexibilización de la práctica de deportes en la mayoría de las patologías, tanto cardíacas como extracardíacas. La evaluación sistematizada del paciente para identificar la presencia de enfermedad CV y estratificar su riesgo permitirá realizar una recomendación individualizada del tipo de actividad física que más beneficios otorgue. En la tabla 3 se adjuntan las principales recomendaciones.

María Victoria Ramos,

https://orcid.org/0000-0002-6349-2781

Este artículo fue aceptado para su publicación por: Editora jefa anterior Dra. María del Pilar Aguilar Passano.

\section{Bibliografía}

1. Pelliccia A, Sharma S, Gati S, Back M, Borjesson M, Caselli S, et al. 2020 ESC Guidelines on sports cardiology and exercise in patients with cardiovascular disease. Eur Heart J. 2020; ehaa605. doi: 10.1093/eurheartj/ehaa605. Online ahead of print. 